\title{
Domains of attraction of walking and running attractors are context dependent: illustration for locomotion on tilted floors
}

\author{
T.D. Frank \\ Department of Psychology, CESPA, University of Connecticut, Storrs, Connecticut, United States \\ E-mail: till.frank@uconn.edu
}

Copyright (C2015 T.D. Frank. This is an open access article distributed under the Creative Commons Attribution License, which permits unrestricted use, distribution, and reproduction in any medium, provided the original work is properly cited.

\begin{abstract}
There is a general consensus that gaits of humans and animals can be considered as spatio-temporal patterns emerging via self-organization. In line with synergetics, a theory of pattern formation and self-organization founded by Haken, an amplitude equation model for human run-walk gait transitions is presented. The model allows for a definition of two distinct attractors representing walking and running. In particular, the size of the two attractors in the space of locomotion speed can be determined as a function of the model parameters. Furthermore, the model parameters can be estimated based on data from walking experiments on treadmills. The approach is illustrated for data reported in the literature on walking experiments involving tilted floors. It is shown that attractor size depends on the floor inclination, which suggests that in general the domains of attraction of walking and running attractors are context dependent. In particular, they depend on floor inclination. In other words, a pattern formation model is presented that describes how sensory feedback about environmental conditions may impact gait control.
\end{abstract}

Keywords: Synergetics, Nonlinear Physics, Gait transitions, Amplitude equations, Order parameters.

\section{Introduction}

At the heart of complex system theory is the theory of pattern formation. Pattern formation is a self-organization process and as a self-organization process has been studied by various research groups and schools [1]. One of these schools is synergetics founded by Haken [2]. While the definition of a pattern is actually based on a mathematical formalism, patterns typically correspond to structures in some appropriately defined space that may evolve over time as well. Spatio-temporal patterns of this kind emerge via bifurcations when certain system parameters, the so-called control parameters, assume critical values [1,2]. One of the most important elements of the theory of pattern formation is that the emergence of patterns can be understood in terms of pattern amplitudes only. That is, the precise details of the patterns play only a limited role in the theory of pattern formation. They primarily affect parameters that in turn affect the evolution of the pattern amplitudes. The evolution equations of the pattern amplitudes correspond usually to coupled ordinary differential equations $[1,2]$. Being somewhat more precise, a pattern formation system typically can feature several different patterns $k=1,2,3, \ldots$ Each pattern exhibits its own amplitude. The observed pattern is a superposition of the individual patterns $k=1,2,3, \ldots$ In particular, if all pattern amplitudes equal zero expect for the amplitude of the pattern $j$, then the observed pattern corresponds 
to the pattern $j$. Likewise, if the pattern formation process exhibits only mutually exclusive patterns, then the amplitudes of the patterns $k=1,2,3, \ldots$ will converge in any case to a so-called winner-takes-all fixed point at which all amplitudes equal zero except for one amplitude. The amplitude equation concept allows a researcher to discuss a given pattern formation problem from a top-down perspective without discussing the details of the patterns under consideration [3].

In synergetics the pattern amplitudes of the emerging patterns are referred to as order parameters. The reason for this is that the amplitudes are considered as counterparts to order parameters of equilibrium phase transitions such as ferromagnetic phase transitions and other order-disorder transitions [2].

The order parameter concept of synergetics for pattern formation has been applied in the field of motor control as well. For example, synchronization of rhythmic limb movements to a metronome beat and synchronizing two oscillating limbs with each other has been modeled using order parameter equations $[4,5,6,7,8]$. Transitions between one-handed and two-handed grasping when the size of the to-be-grasped objects increases have been examined using an order parameter equation $[3,9,10]$. Applications of order parameter equations in social psychology examined infant-mother face-to-face communication [11] and personal space [12] and applications in clinical psychology have addressed schizophrenia, bipolar disorder and obsessive-compulsive-disorder [13, 14, 15, 16, 17, 18, 19].

In the context of gait control, it is of interest to note that walking and running of humans have been considered as spatio-temporal patterns emerging via self-organization of the human motor control system such that the concepts of pattern formation [7] and self-organization [20] apply. Therefore, following the claim made above, walking and running may be studied on the basis of appropriately defined amplitude equations. In fact, for another class of gross motor patterns, namely, grasping patterns, an order parameter model has been developed and applied to experimental data $[3,9,10,21]$. This model can easily be adopted to describe transitions between walking and running in gait transition experiments. However, what has been missing in previous research is an appropriate focus on the role of a particular model parameter: the offset parameter $L_{2,0}$. The parameter $L_{2,0}$ belongs to the second order parameter that describes the amplitude of the second motor pattern (i.e., the running pattern in gait experiments). Therefore, in Sec. 2 the order parameter model developed earlier for grasping transitions $[3,9,10,21]$ will be briefly introduced in the context of gait transitions. Subsequently, an in-depth discussion of the role of the parameter $L_{2,0}$ will be provided. It will be shown that it determines the size of the gait attractors in the space of the locomotion speed. In Sec. 3 the model will be applied to previously reported data from gait transition experiments. Two studies for walking on titled floors will be reviewed and re-analyzed. In these previous studies it has been shown that the speed at which walk-run and run-walk transitions can be observed depends on the inclination angle of the floor. Consequently, the studies support the hypothesis that gait control relies on feedback about the environment. An analysis of this issue with respect to the synergetic gait transition model will shed new light on the nature of this feedback mechanism. It will be shown that the floor inclination affects the aforementioned model parameter $L_{2,0}$. Therefore, the conclusion will be drawn that in general the size of gait attractors is not fixed but context dependent. For example, it is affected by the inclination angle of the supporting surface on which walking or running is performed.

\section{Synergetic gait transition model}

\subsection{Walking and running attractors: definition via amplitude equations}

Let us adopt the grasping transition model developed earlier [9] to describe gait transitions. Note that the grasping transition model is a version of the pattern formation model suggested by Haken [22]. In general, we will consider amplitude equations that have been discussed frequently in the context of pattern formation. The amplitude equations that will be presented below describe pattern formation in fluid layers under Benard instability [22, 23, $24,25,26,27,28,29,30]$, pattern formation in chemical reaction-diffusion systems [31], pattern formation in metal solidification processes [32] and the emergence of patterns in diffusive signaling processes in cells [33, 34]. Moreover, the amplitude equations can be transformed into the form of Lotka-Volterra equations [35]. Such Lotka-Volterra equations have been suggested to capture the amplitude dynamics of neural activity patterns [36]. Therefore, the amplitude equations that will be used below are not only typical for pattern formation systems of the inanimate and animate world, rather, they may reflect neural activity patterns associated with walking and running.

In line with the model on transitions between one-handed and two-handed grasping $[3,9]$, we consider the order 
parameter equations of the form

$$
\begin{aligned}
\frac{\mathrm{d}}{\mathrm{d} t} \xi_{1} & =\lambda_{1} \xi_{1}-g \xi_{2}^{2} \xi_{1}-\xi_{1}^{3}, \\
\frac{\mathrm{d}}{\mathrm{d} t} \xi_{2} & =\lambda_{2} \xi_{2}-g \xi_{1}^{2} \xi_{2}-\xi_{2}^{3} .
\end{aligned}
$$

In Equations (1) and (2), $\xi_{1}$ and $\xi_{2}$ are the order parameters of the walking and running modes, respectively. More precisely, if $\xi_{1}>0, \xi_{2}=0$ holds, then the model describes a walking actor. In contrast, if $\xi_{2}>0$, $\xi_{1}=0$ holds, then the model describes a running actor. In this study, magnitudes of $\xi_{1}$ and $\xi_{2}$ are not directly evaluated. The focus is on examining whether a behavioral mode is present (e.g. $\xi_{1}>0$ ) or absent (e.g. $\left.\xi_{1}=0\right)$. The parameter $g$ is the coefficient that occurs in the mixed terms and represents the strength of the interaction between walking and running. Here, interaction refers to the notion that the two gaits compete with each other for being performed. The larger the interaction between the gaits, the larger is the value of $g$. The parameter $g$ must satisfy $g \geq 1$. Only in this case the model can account for the mutual exclusivity of the behavioral modes. If $g<1$ then modes may co-exist. This is inconsistent with the experimental paradigm under consideration that allows an agent to perform either walking or running but not both at the same time. The parameters $\lambda_{1}$ and $\lambda_{2}$ are growth parameters. If positive they describe how fast the order parameters $\xi_{1}$ and $\xi_{2}$ increase exponentially provided that both parameters are close to zero. The growth parameters also determine the stability of the fixed points, as we will discuss in what follows.

\subsection{Fixed points}

The order parameter equations (1) and (2) exhibit four fixed points provided that $\lambda_{1}, \lambda_{2}>0$. The fixed points are

$$
\begin{aligned}
\xi_{1}=0 & \wedge \xi_{2}=0, \\
\xi_{1}=\sqrt{\lambda_{1}} & \wedge \xi_{2}=0, \\
\xi_{1}=0 & \wedge \xi_{2}=\sqrt{\lambda_{2}}, \\
\xi_{1}>0, \xi_{2}>0 & \wedge \xi_{1}^{2}+\xi_{2}^{2}=\frac{\lambda_{1}+\lambda_{2}}{1+g} .
\end{aligned}
$$

The fixed point $\xi_{1}=0 \wedge \xi_{2}=0$ describes the origin. It is unstable for $\lambda_{1}>0$ or $\lambda_{2}>0$ as can be shown by a linear stability analysis. Since in applications to gait transitions at least one of the two parameters is larger than zero, it follows that the origin is an unstable fixed point. Likewise, the co-existence fixed point defined by Eq. (6) is unstable for $g \geq 1$ as can be shown by a linear stability analysis again [9]. The remaining fixed points defined by Eqs. (4) and (5) are so-called winner-takes-all fixed points. They may both be stable or only one of them may be stable. More precisely, for $\lambda_{1}>\lambda_{2} / g$ the fixed point of the walking mode with $\xi_{1}>0$ is asymptotically stable. Otherwise, the fixed point is unstable. Likewise for $\lambda_{2}>\lambda_{1} / g$ the fixed point of the running mode with $\xi_{2}>0$ is asymptotically stable. Otherwise, the fixed point is unstable. Consequently, for $1 / g<\lambda_{1} / \lambda_{2}<g$ both fixed points are stable.

\subsection{Control parameter}

As reviewed above, on the one hand, $\lambda_{1}$ and $\lambda_{2}$ determine the stability of the two modes: walk and run. On the other hand, in laboratory experiments the locomotion speed is manipulated and gait transitions are observed at certain critical speeds. It is useful to rescale locomotion speed to obtain a dimensionless number, the so-called Froude number [37], which will be denoted by $\alpha$. The Froude number is defined by $\alpha=v^{2} /\left(L_{\text {leg }} g_{\text {grav }}\right)$, where $v$ is the locomotion speed, $L_{\text {leg }}$ is the leg length of the walker and $g_{\text {grav }}$ corresponds to the gravitational constant. Note that the Froude number is defined such that at Froude numbers larger than 1 walking is impossible because the centrifugal force produced by the swinging body will lift a walker off the ground. Only running is possible for Froude numbers $\alpha$ larger than 1. Since according to the model $\lambda_{1}$ and $\lambda_{2}$ affect the stability of walking and running, whereas from experimental studies it is known that $\alpha$ determines the gait transitions, it is assumed that $\lambda_{1}$ and $\lambda_{2}$ depend on $\alpha$. Without loss of generality [9], we assume that the growth parameters are linear functions of the control parameter $\alpha$ like

$\lambda_{1}=1-\alpha$

and

$\lambda_{1}=L_{2,0}+\alpha$. 
In Eq. (7) the constant on the right-hand-side of the equation is chosen such that when $\alpha>1$ we have $\lambda_{1}<0$. In this case, the fixed point that is defined by $\xi_{1}=\sqrt{\lambda_{1}} \wedge \xi_{2}=0$ and represents the walking mode does not exist. Therefore, the choice of the constant is consistent with the biophysical considerations about walking made above. The parameter $L_{2,0}$ occurring in Eq. (8) is an offset parameter. As mentioned in the introduction, we will focus on this parameter and consequently discuss below the meaning of $L_{2,0}$ in detail.

\subsection{Gait transitions}

As mentioned above, the conditions $\lambda_{2}=g \lambda_{1}$ and $\lambda_{1}=g \lambda_{2}$ describe the gait transition points [9]. Solving $\lambda_{2}=L_{2,0}+\alpha=g \lambda_{1}=g(1-\alpha)$ for $\alpha$ we obtain the critical values $\alpha_{c, 2}$ for walk-to-run transitions:

$\alpha_{c, 2}=\frac{g-L_{2,0}}{g+1}$.

In a similar manner, we can derive $\alpha_{c, 1}$ for run-to-walk transitions like

$\alpha_{c, 1}=\frac{1-g L_{2,0}}{g+1}$.

That is, the fixed point $\xi_{1}=0 \wedge \xi_{2}=\sqrt{\lambda_{2}}$ (representing running) is asymptotically stable for $\alpha>\alpha_{c, 1}$ and unstable for $\alpha<\alpha_{c, 1}$. Likewise, the fixed point $\xi_{1}=\sqrt{\lambda_{1}} \wedge \xi_{2}=0$ (representing walking) is asymptotically stable for $\alpha<\alpha_{c, 2}$ and unstable for $\alpha>\alpha_{c, 2}$. Consequently, when increasing locomotion speed $v$ and the Froude number $\alpha$ gradually (ascending experimental condition) the walk-to-run transition occurs at $\alpha=\alpha_{c, 2}$. In contrast, when decreasing locomotion speed $v$ and the Froude number $\alpha$ gradually (descending experimental condition) the run-to-walk transition occurs at $\alpha=\alpha_{c, 1}$.

\subsection{Hysteresis}

In the context of gait transitions, note that $\alpha_{c, 2} \geq \alpha_{c, 1}$ (because we consider the case $g \geq 1$ ). That is, the model predicts hysteretic transitions [9]. In particular, combining Eqs. (9) and (10), we compute the hysteresis size $\Delta \alpha=\alpha_{c, 2}-\alpha_{c, 1}$ by

$\Delta \alpha=\frac{(g-1)\left(1-L_{2,0}\right)}{1+g}$.

In the hysteresis interval given by $\left[\alpha_{c, 1}, \alpha_{c, 2}\right]$ the system is bistable. Since the function $u(g)=(g-1) /(g+1)$ is a monotonically increasing function for $g \geq 1$ starting at $u(g=1)=0$, from Eq. (11) it follows that the larger the interaction parameter $g$ is, the wider is the bistable domain (see also Fig. 4a in Ref. [3])

\subsection{Parameter estimation}

From Eqs. (9) and (10) it follows that the offset parameter $L_{2,0}$ can be computed like [9]

$L_{2,0}=1-\alpha_{c, 1}-\alpha_{c, 2}$.

Moreover, if we substitute Eq. (12) into Eq. (9) and solve for $g$, we obtain

$g=\frac{1-\alpha_{c, 1}}{1-\alpha_{c, 2}}$.

Equations (12) and (13) are the estimators for the model parameters $L_{2,0}$ and $g$. Let us assume that in gait transition experiments the critical Froude numbers $\alpha_{c, 1}$ and $\alpha_{c, 2}$ can be measured. Then based on the experimental values for $\alpha_{c, 1}$ and $\alpha_{c, 2}$, the model parameters $L_{2,0}$ and $g$ can be computed using Eqs. (12) and (13).

\subsection{The role of $L_{2,0}$ for the size of gait attractors in the space of locomotion speed}

As mentioned above, in order to study walk-to-run and run-to-walk transitions, the Froude number $\alpha=v^{2} /\left(L_{\operatorname{leg}} g_{\text {grav }}\right)$ seems to be a promising control parameter because it predicts that any walker must transition to running at $\alpha=1$ irrespective of the leg length of the walker. 
Keeping this argument in mind, we will discuss the meaning of the model parameter $L_{2,0}$. In previous studies on grasping transitions [3], the parameter $L_{2,0}$ has been interpreted as a overall measure for the "strength" of the second behavioral mode (which is the two-handed grasping in the context of grasping experiments or running in the context of gait transition experiments). More precisely, $L_{2,0}$ determines the overall magnitude of the growth parameter $\lambda_{2}$ of the second mode. This is illustrated in Fig. 1. When $L_{2,0}$ increases then the growth parameter of the second mode becomes stronger for any given Froude number $\alpha$. More precisely, the function $\lambda_{2}(\alpha)$ is shifted to higher values (see Fig. 1 again).

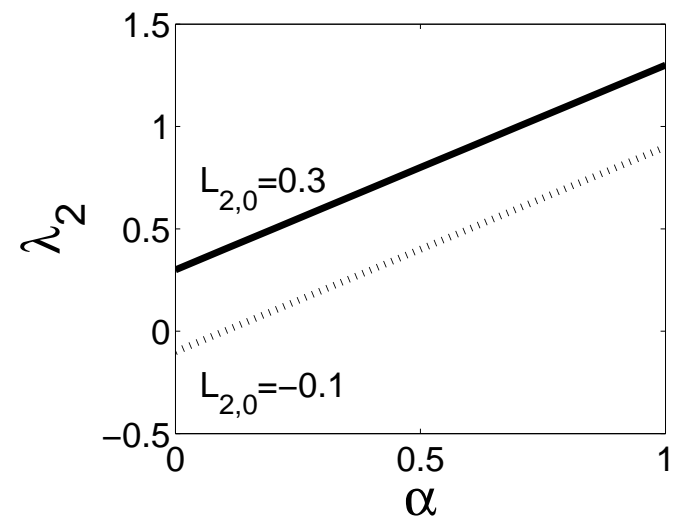

Figure 1: Growth parameter $\lambda_{2}$ as a function of $\alpha$ for two different offset parameters $L_{2,0}=-0.1$ (dashed line) and $L_{2,0}=0.3$ (solid line) as computed from Eq. (8).

Although this observation captures an important aspect of $L_{2,0}$, it does not refer directly to the bifurcation points $\alpha_{c, 1}$ and $\alpha_{c, 2}$ of gait transitions as defined by Eqs. (9) and (10). From Eqs. (9) and (10) it follows that the critical values $\alpha_{c, 1}$ and $\alpha_{c, 2}$ decay as functions of $L_{2,0}$. However, this affects the domains of the attraction for the walking and running attractors differently. As shown in Fig. 2 the domain of the walking attractor in the space of locomotion speed measured in terms of Froude numbers $\alpha$ is given by the Froude numbers $\alpha$ smaller than the boundary $\alpha_{c, 2}=q\left(g-L_{2,0}\right)$ with $q=1 /(1+g)$. In contrast, the domain of the running attractor measured in the space of Froude numbers $\alpha$ is given by the Froude numbers $\alpha$ larger than the boundary $\alpha_{c, 1}=q\left(1-g L_{2,0}\right)$. If $L_{2,0}$ increases then both boundaries $\alpha_{c, 1}$ and $\alpha_{c, 2}$ are shifted to lower values. This implies that the domain for the walking attractor becomes smaller, whereas the domain for the running attractor becomes larger. That is, $L_{2,0}$ reflects the dominance of the running attractor relative to the walking attractor, in the sense that increasing $L_{2,0}$ increases the dominance of the running attractor over the walking attractor in the space of the relative locomotion speeds $\alpha$.

Let us make this interpretation of $L_{2,0}$ more quantitative. To this end, we introduce the size of the domain of an attractor in the space given by the interval $\alpha=[0,1]$. Consequently, the size $S$ reflects a percentage value ranging from 0 to 100 percent. From Fig. 2 it follows that the size of the walking attractor is defined by

$S($ walk $)=\left\{\begin{array}{ccc}1 & \text { for } & L_{2,0}<-1 \\ \alpha_{c, 2} & \text { otherwise } & \\ 0 & \text { for } & L_{2,0}>g\end{array}\right.$

Likewise, the size of the running attractor is defined by

$S($ run $)=\left\{\begin{array}{ccc}1 & \text { for } & L_{2,0}>1 / g \\ 1-\alpha_{c, 1} & \text { otherwise } & \\ 0 & \text { for } & L_{2,0}<-1\end{array}\right.$

Figure 3 depicts $S($ walk $)$ and $S($ run $)$ as functions of $L_{2,0}$ for a given parameter $g$. Again, we see that the two gait attractors are affected differently by $L_{2,0}$. The size $S\left(\right.$ walk) of the walking attractor decays when $L_{2,0}$ is increased. In contrast, the size of the running attractor $S($ run $)$ is increased when $L_{2,0}$ is increased. Note that for any parameter $g \geq 1$ the graphs $S($ walk) and $S($ run $)$ behave qualitatively as shown in Fig. 3 . 


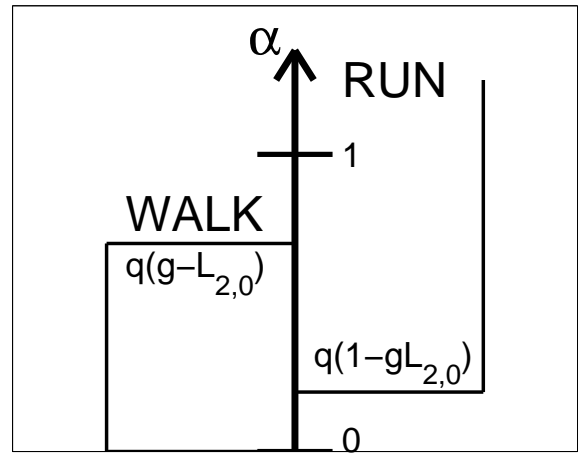

Figure 2: Domains of attraction of walking and running attractors in the space of relative locomotion speed measured in terms of $\alpha$. There are two relevant boundary levels: $\alpha_{c, 2}=q\left(q-L_{2,0}\right)$ for walk (see left) and $\alpha_{c, 1}=q\left(1-L_{2,0}\right)$ for run (see right). Here: $q=1 /(1+g)$.

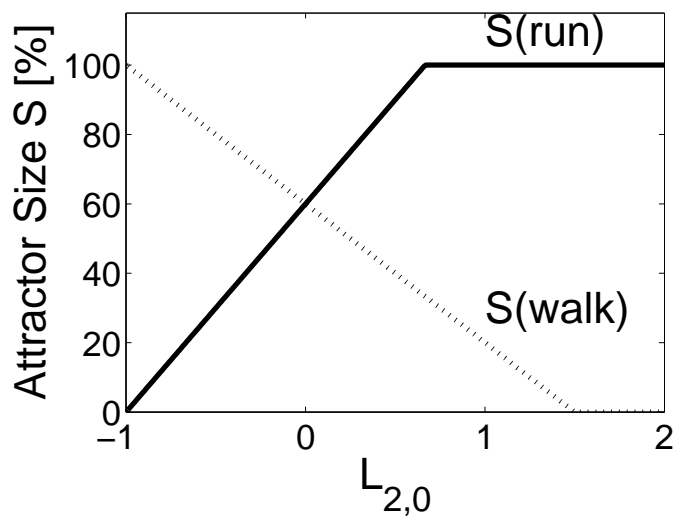

Figure 3: Size of gait attractors for walking (dotted line) and running (solid line) as a function of $L_{2,0}$. The graphs were computed from Eqs. (14) and (15). Parameter: $g=1.5$.

\section{Gait transition experiments on tilted floors}

Let us review two studies in which gait transitions on tilted floors have been observed [38, 39]. In both studies participants were asked to walk on a treadmill. Treadmill speed was either gradually increased to induce a walkto-run transition or gradually decreased to induce a run-to-walk transition. Importantly, both studies allow us to discuss gait transitions with respect to relative locomotion speed as defined by the Froude number $\alpha$. In both studies, the ramp of the treadmill was tilted such that walkers had to walk or run on a tilted floor. In the study by Hreljac et al. [39] three angles of inclination were used. For sake of conveniency, the inclination angles were express in percentage values like $\theta=0 \%, \theta=10 \%$, and $\theta=15 \%$. In the study by Diedrich and Warren [38] two angles of inclination were used: $\theta=0 \%$ and $\theta=10 \%$ (the latter was equivalent to an angle of 5.7 degrees).

\subsection{Descriptive results}

Table 1 shows for the three angles $\theta$ the critical treadmill speeds at which on the average walk-to-run and run-towalk transitions were observed by Hreljac et al. [39]. Furthermore, since Hreljac et al. reported a mean leg length $L_{\text {leg }}$ of $0.88 \mathrm{~m}$, the critical locomotion speeds $v_{c, 1}$ and $v_{c, 2}$ were transformed into Froude numbers $\alpha_{c, 1}$ and $\alpha_{c, 2}$. Overall the critical velocities (locomotion speeds) decreased when the inclination of the floor was increased. This implies that the critical Froude numbers calculated in Table 1 decreased as well as a function of $\theta$.

In the study by Diedrich and Warren [38], mean critical Froude values averaged across walk-to-run and run-to- 
Table 1: Descriptive measures observed in the gait transition experiment by Hreljac et al. [39]. Critical Froude values were not reported in Hreljac et al. [39]. The critical Froude numbers shown in this table were calculated from the velocities using $\alpha_{c}=v_{c}^{2} /\left(L_{\text {leg }} g_{\text {grav }}\right)$ with $L_{\text {leg }}=0.88 \mathrm{~m}$.

\begin{tabular}{llcc}
\hline$\theta[\%]$ & Condition & Critical velocities $v_{c}[\mathrm{~m} / \mathrm{s}]$ & Critical Froude values $\alpha_{c}$ \\
\hline 0 & Walk-to-run & 1.9 & $\alpha_{c, 2}=0.42$ \\
& Run-to-walk & 1.8 & $\alpha_{c, 1}=0.38$ \\
10 & Walk-to-run & 1.8 & $\alpha_{c, 2}=0.38$ \\
& Run-to-walk & 1.7 & $\alpha_{c, 1}=0.33$ \\
\multirow{2}{*}{15} & Walk-to-run & 1.7 & $\alpha_{c, 2}=0.33$ \\
& Run-to-walk & 1.6 & $\alpha_{c, 1}=0.30$ \\
\hline
\end{tabular}

walk transitions were reported. Mean critical Froude values of $\alpha_{c, m}=0.55$ for $\theta=0 \%$ and $\alpha_{c, m}=0.46$ for $\theta=10 \%$ were observed. Again, it was observed that the critical Froude values on average shifted to lower values when the inclination of the floor was increased.

\subsection{Model-based re-analysis of gait transition experiments}

Using Eqs. (12) and (13), we calculated the model parameters $g$ and $L_{2,0}$ from the critical Froude values $\alpha_{c, 1}$ and $\alpha_{c, 2}$ reported in Table 1 . Table 2 reports the estimated model parameters thus obtain. The interaction parameter $g$ varied only slightly as a function of the inclination angle. In contrast, $L_{2,0}$ increased with increasing angle $\theta$ by a fair amount. In line with our considerations on $L_{2,0}$ made earlier, we conclude that the dominance of the running attractor increased when inclination angle was increased. This model-based conclusion is illustrated in Fig. 4. Figure 4 plots the domains of attraction for the walking and running attractors in the space of relative locomotion speeds (as measured by $\alpha$ ). The domains are plotted for the three inclination angles separately. The graphical illustration supports our conclusion that the domain of attraction of the running attractor increased with increasing inclination angle. In contrast, the domain of attraction of the walking attractor decreased with increasing inclination angle. Finally, using Eqs. (14) and (15) attractor size was determined. The results are reported in Table 3. In line with the increase of $L_{2,0}$ as a function of $\theta$, we found that the size $S($ walk) of the walking attractor decreased when increasing the floor inclination $\theta$, while the size $S($ run $)$ of the running attractor increased when increasing $\theta$.

Table 2: Model parameters $g$ and $L_{2,0}$ estimated for the gait transitions observed in the gait transition experiment by Hreljac et al. [39].

\begin{tabular}{lcc}
\hline$\theta[\%]$ & $g$ & $L_{2,0}$ \\
\hline 0 & 1.07 & 0.21 \\
10 & 1.06 & 0.29 \\
15 & 1.06 & 0.37 \\
\hline
\end{tabular}

Table 3: Effect of floor inclination on the size of the gait attractors for walking ( $($ walk $))$ and running $(S($ run $))$ as obtained from the model-based re-analysis of the gait transition study by Hreljac et al. [39].

\begin{tabular}{lcc}
\hline$\theta[\%]$ & $S($ walk $)[\%]$ & $S($ run $)[\%]$ \\
\hline 0 & 42 & 62 \\
10 & 38 & 67 \\
15 & 33 & 70 \\
\hline
\end{tabular}

As mentioned above, in the study by Diedrich and Warren [38] only critical Froude values averaged across walk-to-run and run-to-walk transitions were reported. It is known that such critical values are consistent with the critical values obtained from random conditions in behavioral transition experiments [40]. However, such random conditions cannot address hysteresis. Therefore, for these averaged critical Froude values (reflecting a pseudorandom condition) it is useful to put the interaction parameter $g$ equal to unity. In this case, from Eq. (11) it follows that the model does not account for any hysteresis effect. That is, for $g=1$ hysteresis size equals zero. 


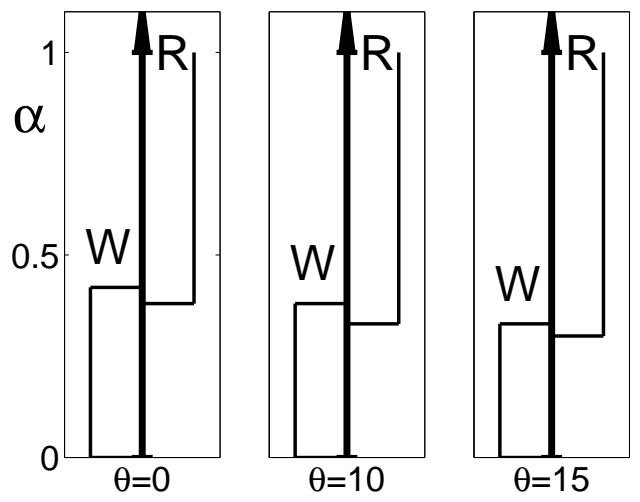

Figure 4: Domains of attraction for walking and running attractors as functions of the floor inclination. See text for details.

Re-writing Eq. (12) like $L_{2,0}=1-2 \alpha_{c, m}$, where $\alpha_{c, m}$ is the averaged critical Froude number $\alpha_{c, m}=\left(\alpha_{c, 1}+\alpha_{c, 2}\right) / 2$, we estimated $L_{2,0}$ from the values $\alpha_{c, m}$ reported in the study by Diedrich and Warren [38]. From $\alpha_{c, m}=0.55$ for $\theta=0 \%$ we obtain $L_{2,0}=-0.10$. From $\alpha_{c, m}=0.46$ for $\theta=10 \%$ we obtain $L_{2,0}=0.08$. Just as in the previous study, we found that $L_{2,0}$ increased when floor inclination was increased.

\section{Discussion}

We studied gait transitions between walking and running from a nonlinear physics perspective and within the framework of pattern formation. To this end, we presented a re-interpretation of a previously developed model for grasping transitions. The model describes on a macro-level two order parameters $\xi_{1}$ and $\xi_{2}$ reflecting the amplitudes of the walking and running gait patterns. We demonstrated that the model can capture hysteretic gait transitions from walk-to-run and run-to-walk in terms of bifurcations involving the order parameters $\xi_{1}$ and $\xi_{2}$. The model features two parameters: $g$ and $L_{2,0}$. Importantly, based on experimentally observed data both model parameters can be estimated.

In this context, we arrived at a novel interpretation of the model parameter $L_{2,0} . L_{2,0}$ determines the dominance of the running attractor relative to the walking attractor. The domain of attraction in the space of relative locomotion speeds is positively correlated with $L_{2,0}$ for the running attractor but negatively correlated with $L_{2,0}$ for the walking attractor. Likewise, using an appropriately defined measure for the attractor size $S$ we showed that attractor size $S$ increases with $L_{2,0}$ for the running attractor but $S$ decreases with $L_{2,0}$ for the walking attractor.

Two experiments reported in the literature on the impact of floor inclination were considered. The critical Froude numbers decreased with higher level of inclination. The first model parameter $g$ varied only slightly with the inclination level meaning that the interaction between the walking and running modes remained largely unaffected by the manipulation of the floor inclination. In contrast, the parameter $L_{2,0}$ increased by a fair amount with the inclination level demonstrating that the attractor domains in general and attractor size in particular are affected by floor inclination. This illustrates that external conditions, that is, environmental conditions, in general have an impact on the self-organization of human gaits and can affect gait attractors in particular. In other words, our model-based analysis suggests that the gait attractor involved in human locomotion are context dependent.

\section{References}

[1] G. Nicolis, Introduction to nonlinear sciences, Cambridge University Press, Cambridge, 1995.

[2] H. Haken, Synergetics. An introduction, Springer, Berlin, 1977.

[3] S. M. Lopresti-Goodman, M. T. Turvey, T. D. Frank, Behavioral dynamics of the affordance "graspable", Attention, Perception, and Psychophysics 73 (2011) 1948-1965.

[4] P. G. Amazeen, E. Amazeen, M. T. Turvey, Dynamics of human intersegmental coordination: theory and research, in: D. A. Rosenbaum, C. E. Collyer (Eds.), Timing of behavior, MIT Press, Cambridge, 1998, pp. 237-259. 
[5] P. J. Beek, C. E. Peper, D. F. Stegeman, Dynamical models of movement coordination, Hum. Movement Sci. 14 (1995) 573-608.

[6] J. A. S. Kelso, Dynamic patterns - The self-organization of brain and behavior, MIT Press, Cambridge, 1995.

[7] H. Haken, Principles of brain functioning, Springer, Berlin, 1996.

[8] T. D. Frank, P. L. Silva, M. T. Turvey, Symmetry axiom of the Haken-Kelso-Bunz coordination dynamics revisited in the context of cognitive activity, Journal of Mathematical Psychology 56 (2012) 149-165.

[9] T. D. Frank, M. J. Richardson, S. M. Lopresti-Goodman, M. T. Turvey, Order parameter dynamics of body-scaled hysteresis and mode transitions in grasping behavior, J. Biol. Phys. 35 (2009) 127-147.

[10] S. M. Lopresti-Goodman, M. T. Turvey, T. D. Frank, Negative hysteresis in the behavioral dynamics of the affordance "graspable", Attention, Perception, and Psychophysics 75 (2013) 1075-1091.

[11] T. D. Frank, A nonlinear physics model based on extended synergetics for the flow of infant actions during infant-mother face-to-face communication, International Journal of Scientific World 2 (2014) 62-74.

[12] T. D. Frank, Interpersonal distances are the consequence of self-organization of human spatial behavior: a theoretical study based on synergetics, Universal Journal in Psychology 2 (2014) 285-289.

[13] H. Haken, G. Schiepek, Synergetik in der Psychologie, Hogrefe, Gottingen, 2006, in German.

[14] W. Tschacher, J. P. Dauwalder, Situated cognition, ecological perception, and synergetics: a novel perspective for cognitive psychology?, in: W. Tschacher, J. P. Dauwalder (Eds.), Dynamics, synergetics, autonomous agents, World Scientific, Singapore, 1999, pp. 83-104.

[15] T. D. Frank, Multistable perception in schizophrenia: a model-based analysis via coarse-grained order parameter dynamics and a comment on the 4th law, Universal Journal in Psychology 2 (2014) 231-240.

[16] T. D. Frank, A limit cycle model for cycling mood variations of bipolar disorder patients derived from cellular biochemical reaction equations, Communications in Nonlinear Science and Numerical Simulation 18 (2013) 2107-2119.

[17] T. D. Frank, From systems biology to systems theory of bipolar disorder, in: F. Miranda (Ed.), Systems theory: perspectives, applications and developments, Nova Publ., New York, 2014, pp. Chap. 2 (pp. 17-36).

[18] T. D. Frank, Action flow in obsessive-compulsive disorder rituals: a model based on extended synergetics and a comment on the 4th law, Journal of Advances in Physics 5 (2014) 845-853.

[19] T. D. Frank, Secondary bifurcations in a Lotka-Volterra model for n competitors with applications to action selection and compulsive behaviors, Int. J. Bif. and Chaos 24 (2014) article 1450156.

[20] F. J. Diedrich, W. H. Warren, Why change gaits? Dynamics of the walk-run transition, J. Exp. Psychol. - Hum. Percept. Perform. 21 (1995) 183-202.

[21] T. D. Frank, J. van der Kamp, G. J. P. Savelsbergh, On a multistable dynamic model of behavioral and perceptual infant development, Dev. Psychobiol. 52 (2010) 352-371.

[22] H. Haken, Synergetic computers and cognition, Springer, Berlin, 1991.

[23] M. Bestehorn, R. Friedrich, H. Haken, Two-dimensional traveling wave patterns in nonequilibrium systems, Z. Physik B 75 (1989) 265-274.

[24] M. Bestehorn, R. Friedrich, H. Haken, Modulating traveling waves in nonequilibrium systems: the blinking state, Z. Physik B 77 (1989) 151-155.

[25] M. Bestehorn, H. Haken, Associative memory of a dynamical system: an example of the convection instability, Z. Physik B 82 (1991) 305-308.

[26] T. D. Frank, Multistable pattern formation systems: candidates for physical intelligence, Ecological Psychology 24 (2012) 220-240.

[27] J. Swift, P. Hohenberg, Hydrodynamic fluctuations at the convective instability, Phys. Rev. A 15 (1977) $319-328$.

[28] A. C. Newell, J. A. Whitehead, Finite bandwidth, finite amplitude convection, J. Fluid Mech. 38 (1969) $279-303$.

[29] M. C. Cross, P. C. Hohenberg, Pattern formation outside of equilibrium, Rev. Mod. Phys. 65 (1993) 851-1112. 
[30] C. A. Jones, M. R. E. Proctor, Strong spatial resonance and traveling waves in Benard convection, Phys. Lett. A 121 (1987) 224-228.

[31] D. J. Wollkind, L. E. Stephenson, Chemical Turing pattern formation analyses: comparison of theory with experiment, SIAM Journal of Applied Mathematics 61 (2000) 387-431.

[32] H. Levine, Directional solidification: theoretical models and current understanding, in: D. Walgraef, N. M. Ghoniem (Eds.), Patterns, defects, and material instabilities, Kluwer Academic Publishers, Dordrecht, 1990, pp. 123-133.

[33] C. Rattanakul, Y. Lenbury, D. J. Wollkind, V. Chatsudthipong, Weakly nonlinear analysis of a model of signal transduction pathway, Nonlinear Analysis 71 (2009) e1620-e1625.

[34] L. E. Stephenson, D. J. Wollkind, V. Chatsudthipong, Weakly nonlinear analysis of one-dimensional Turing pattern formation in activator-inhibitor/immobilizer model system, Journal of Mathematical Biology 33 (2009) 771-815.

[35] T. D. Frank, Psycho-thermodynamics of priming, recognition latencies, retrieval-induced forgetting, priming-induced recognition failures and psychopathological perception, in: N. Hsu, Z. Schütt (Eds.), Psychology of priming, Nova Publ., New York, 2012, pp. Chap. 9 (pp. 175-204).

[36] M. I. Rabinovich, M. K. Muezzinoghu, I. Strigo, A. Bystritsky, Dynamic principles of emotion-cognition interaction: mathematical images of mental disorders, PLoS ONE 5 (2010) e12547.

[37] R. M. Alexander, Introduction to the theory of ferromagnetism, New York, W. H. Freeman, 1992.

[38] F. J. Diedrich, W. H. Warren, The dynamics of gait transitions: effects of grade and load, J. Motor Behav. 30 (1998) 60-78.

[39] A. Hreljac, R. Imamura, R. F. Escamilla, W. B. Edwards, Effects of changing protocol, grade, and direction on the preferred gait transition speed during human locomotion, Gait \& Posture 25 (2007) 419-424.

[40] M. J. Richardson, K. L. Marsh, R. M. Baron, Judging and actualizing intrapersonal and interpresonal affordances, J. Exp. Psychol. - Hum. Percept. Perform. 33 (2007) 845-859. 